\title{
Making to Measure? Re-Considering Assessment in Professional Continuing Education
}

Tara Fenwick

Studies in Continuing Education

\begin{abstract}
Drawing on studies of teachers, accountants and pharmacists conducted in Canada, this essay examines models for assessing professional learning that currently enjoy widespread use in continuing education. These models include professional growth plans, self-administered tests, and learning logs, and they are often used for regulatory as well as developmental purposes by professional associations. The essay argues what others have critiqued about such self-assessment models: that their assumptions about learning are problematic and limiting in a number of respects, privileging human consciousness and intention, and literally 'making' a particular professional subject that is atomised and conservative. The essay goes on to suggest alternative perspectives that are receiving increasing attention in theorising work-related learning and that may offer fruitful questions for re-considering the nature of professional learning and its assessment. Three perspectives in particular are outlined, all of which shift the focus from the learning subject to practice as material, emergent and systemic: complexity theory, actor-network theory and cultural-historical activity theory. The discussion concludes with possible approaches to assessment of professional practice suggested by these perspectives.
\end{abstract}

\section{Introduction}

In many professions, policies to mandate and measure lifelong learning or 'professional development' (PD) are becoming increasingly common. These policies are usually linked to licensure, for example by requiring members of a professional association to submit annual reports of learning or statements of hours spent participating in activities designated as learning in order to maintain their professional certification. The justification for such policies is embedded in what Evetts (2003) shows to be traditional conceptions of the professional as autonomous, self-regulating, with specialised expertise and responsibility to the public to maintain particular standards in this expertise. The latter has become particularly important at a time of increased public scrutiny and demands for explicit accountability. For example, the policy for Continuous Professional Learning and Development for certified management accountants in Alberta reflects sentiments found throughout professions:

CMAs hold positions of utmost trust in organizations and, as such, owe a professional standard of care to employers, clients, fellow members and the public at large.

By formalizing our commitment to the principles of ongoing professional development, CMA Alberta members uphold the trust of all stakeholders, further solidifying our reputation as the designation of choice amongst strategic financial management professionals. Competence and Proficiency are an important component of the CMA Alberta Code of Ethics. (CMA, 2000)

The questions that challenge many professional associations are, How can assessment measure a professional's relative level of competency in ways that can reliably maintain certification, and 
inform provision of continuing professional development? How can the professional's actual learning that occurs in continuing development activities be measured? These questions about evaluating a professional's knowledge emerge from certain assumptions. First, that learning is an individual affair of acquiring new knowledge and skills. Second, that participation in special activities designated or designed for professional development will produce this learning. Third, that it is desirable to pre-determine and to regulate the knowledge most worthwhile for a professional to learn regardless of constantly shifting contexts of practice. Other assumptions accrue to notions of professional autonomy and self-regulation, in particular that self-assessment is a useful method to both measure lifelong learning and promote professionals' motivation to engage in PD activity.

This discussion argues that these assumptions can be problematic, constructing a conception of knowledge and practice that 'makes' what it purports to measure. They also can lead to unhelpful questions about professionals' learning processes, and how they may be fostered and assessed. Further, as many have argued, such assumptions limit and manage the professional, disciplining a universal professional subject that conforms to neo-liberal norms: continuously self-assessing and self-perfecting, enterprising, mobile and flexible (McWilliam, 2002; Stronach et al., 2002). This atomised subject seeks to adapt to institutions of governance and knowledge rather than transforming them, and is focused on performing correctly rather than challenging norms of performance. To interrupt and expand these individualistic, acquisitive, psychologised and mentalist assumptions about learning, some educationists have been exploring socio-material perspectives - showing how practice and knowledge emerges through relations among objects as well as people in joint action (e.g. Davis and Sumara, 2006; Fenwick and Edwards, forthcoming; Fox, 2005; Oberg and Biesta, 2007; Sawchuk et al, 2006). These perspectives conceptualise professional learning as unpredictable, as rooted not in individual heads or bodies but in provisional networks of people, activity, objects and technology, and that is expansive rather than acquisitive. These assumptions pose questions about assessment that in turn may suggest approaches to help trace the complexities and contradictions of today's professional practice. The argument is not that the only forms of professional knowledge in practice are distributed and embodied. Clearly, as Eraut $(1994,2004)$ has shown, professional expertise involves complex personal modes of cognition, meta-cognition, intuiting, relating, and mediating knowledge, people and artefacts. The argument here is that our predominant conceptions guiding learning assessment often fail to recognize aspects of practice and knowing that clearly are distributed, emergent, and rooted in material as well as social connections.

The discussion begins with examples of approaches used to assess continuing learning in Canadian professional associations of accountants, pharmacists and teachers. Problems as well as benefits are shown in these approaches relative to what is being measured and what these measures 'make' through particular conceptions of professional knowledge. Then a brief overview is provided of relational and system-oriented learning perspectives offered by culturalhistorical activity theory, actor-network theory, and complexity theory. While each of these perspectives opens new possibilities for understanding and even assessing professional learning, they each also have their own limitations. Viewing them alongside the currently pervasive individualistic models is not intended to present them as superior, but as offering alternative conceptions and questions about professional learning and assessment that might interrupt and open prevailing assumptions. 


\section{Assessment of Lifelong Learning in Professional Practice}

The following examples emerged from research conducted with professional associations in Alberta, Canada since 2000. In Canada, professional regulation is under provincial jurisdiction, with different provinces exercising unique certification requirements, assessments and PD provisions. In each story here, the professional association implemented a policy to mandate and then monitor professional learning largely relying upon self-assessment. Each association adopted a different approach, with its own advantages and disadvantages. However as the brief discussion following the examples will show, the assessment methods are consistent in their general assumptions about learning and assessment, and in the problems encountered.

\section{Teachers: Teacher professional growth plans}

To ensure the continuous lifelong learning of its public school teachers, the Alberta policy 2.1.5 Teacher Growth, Supervision and Evaluation (Alberta Learning, 1998) requires all Alberta teachers to create and maintain a 'teacher professional growth plan' (TPGP). This plan must contain at least three goals and an action plan for professional development, reviewed annually with a supervisor to examine how the plan met the goals in terms of teacher learning. The policy appears to support all the good things that teacher development literature has been advocating: it promotes reflective practice, explicitly values teachers' learning, and works from a positive model of teachers as responsible professionals, self-directed continuous learners - rather than a negative deficit model. Any concerns about accountability or teacher quality that may be levelled against school districts can be answered by pointing to the existence of annual written records maintained by every teacher, reporting areas of practice requiring improvement, and describing specific actions taken to address these areas.

A qualitative study of 2000-2002 examined implementation of these professional growth plans and compared teachers' and administrators' meanings and usages of TPGPs within and across school districts (Fenwick 2003). Highest satisfaction was evident in schools where time, support, flexibility and resources had been allocated to the growth plans, and where a culture valuing reflective practice was already in place. Some teachers actually reported an increase in their participation in specific PD activities. Many noted a clearer purpose and focus on what was important to learn when using their own goals as a barometer, and felt affirmation in the written evidence of their own skill changes: 'You get lost in your everyday stuff and not realise how much you do accomplish in a year. . . . success is often very small and it takes an extreme length of time before you see a real change. [The TPGP] gives you concrete examples of what you have accomplished' (Fenwick, 2003). In some schools TPGPs were used as a stimulus for gathering teachers in dialogue. Teachers found this new collaborative space the most valuable element of the TPGP process for sharing affirmations, strategies and dilemmas about professional practice and its representation.

The disadvantages of TPGPs were also evident in every context. All interviewees noted how time-consuming the growth plans were for both teachers and their supervisors if engaged meaningfully. Trust and risk issues were uppermost as teachers positioned themselves carefully in what professional 'weaknesses' or 'growth areas' they would reveal to a supervisor as a learning goal. Some were concerned about the emphasis on potentially reductionist visible outcomes; others used this for easy subversion, writing down a few technical goals that the district valued that year such as learning power point software. The linear process of learning as 
goal-setting was problematic because teacher learning is more fluid and unpredictable than the one-year TPGP process of plan-action-measure allows: 'Some goals just aren't set-able in September' said one teacher (Fenwick, 2003). Teachers and supervisors both were concerned that TPGPs focused on observable indicators, foregrounding teaching technique and educational strategies for growth (such as workshops and conferences) and overshadowing more intangible, complex processes of learning in practice. Some wondered whether written goals inhibited the spontaneity of following unforeseen opportunities. Teachers also wondered: Did I fail if my goal wasn't completed? Is a goal ever completely finished? For administrators, there was concern about 'what counts' as a professional learning goal, and what were the limits of 'appropriate' goals for professional growth. While some liked the TPGP move away from a deficit model, others were concerned that 'marginal' teaching was not being addressed effectively. Several wondered how individual teachers could identify areas they genuinely needed to improve without external assistance.

\section{Pharmacists: Self-assessment checklists}

With new emphases on lifelong learning, increased public concern over dispensing errors, and changes to practice (such as pharmacists being granted to authority to prescribe pharmaceuticals), the Alberta College of Pharmacists developed for its members a registration requirement of mandatory professional learning and selfassessment. Most other provincial colleges of pharmacy in Canada undertook similar initiatives. An extensive competency profile was created, and subsequently a 'continuing competence' procedure was implemented (Alberta College of Pharmacists, 2008). This procedure offers online tools for members to complete a self-assessment, an annual rating of their level of knowledge and skill in a lengthy and detailed list of competencies, a learning plan, as well as a 'continuing professional development learning log'.

Individuals are required to complete a minimum of 15 hours of annual continuing education, and record these learning activities in a log that asks for the relation of the activity to a specific approved competency. Traditional PD consisted of text modules providing pages of instruction in specific topics such as anti-coagulation management or diabetes followed by self-administered multiple choice tests of information recall.

Supplementing these procedures is a system of on-site practice review, whereby registered pharmacists are selected at random and visited by observers who score their practice using detailed checklists.

A number of problems with this assessment system were identified by administrative personnel in both Continuing Pharmacy Education (CPE) and the College. First was the concern about relying upon self-assessment to ensure pharmacists' competency and protection of public safety. The annual submissions revealed little about the depth of thought or the accuracy of judgment exercised by individuals in considering each competency and rating their own strength of understanding. Assessments of competency lists did not capture the complexity of pharmacists' knowledge, or their knowledge-in-use through problem solving in specific contexts. Module tests ultimately relied upon test-taker's honesty. As the CPE Director noted, the exercise can easily turn into ticking a series of boxes: 'I do this, I know that...' (personal communication, 2008). A professional development course was created to help individuals understand reflective inquiry and the purposes of self-assessment, although it was suspected that most professionals who elected to take the course were already 'converts'. The challenge articulated by the College and CPE staff was how to help individual practitioners to develop awareness of their own 'blind 
spots' of practice, particularly in areas where they had less competency, so that they could seek out the information and resources they needed to solve a problem. This issue, documented in other contexts (Austin et al., 2008) has opened the question about whether these assessments require validation with external assessment and with demonstration of actual outcomes of work.

All of this led to the second major problem protested most often by practitioners: time. In the current period of pharmacist shortages, those in the field experience long shifts, managerial quotas, and general conditions of moment-to-moment survival that make tools of 'reflective practice' impossible for some to envision. Its protocols presented a new layer of work with doubtful utility. Furthermore, these assessment approaches emphasised individualised isolation, deficit, and codes of knowledge that neither corresponded with nor affirmed the actuality of everyday practice and its challenges. Finally, according to the CPE Director, written reflection for many pharmacists imposes a modality and structure of knowledge that does not fit their everyday dilemmas mixing pharmacology with problem-solving, diagnosing, and managerial activities.

\section{Certified management accountants (CMAs): Learning event logs}

A policy introduced for CMAs in Alberta in 2000 requires that all certified members must participate in a minimum of 120 hours of professional learning activity over a three-year period, with a minimum of 30 hours annually towards the 120 (CMA, 2000). In this policy, the professional Association declares itself to be 'very flexible' in identifying a range of activities that may be declared. 'Verifiable' activities include conferences with receipts, presentations with slides, courses with reportable grades or certificates, while non-verifiable activities that may be reported include reading professional journals, doing web-based research, and mentoring others. Members must retain a log and evidential documentation of these activities, and submit to the association an 'annual declaration' indicating compliance with the policy along with their annual membership fee. The Association may 'audit' a member at any time to check the logs of specific activity participation. In choosing activities that the Association deems relevant to professional learning, CMAs are encouraged to use the CMA Competency Map as a guide. This map presents six 'functional' competencies for the professional CMA (strategic management, risk management and governance, performance management, performance measurement, financial resource management, and financial reporting) and four 'enabling' competencies (problem solving and decision making, leadership and group dynamics, professionalism and ethical behavior, and communication).

This model of recording learning events appears to be beneficial in signalling the value accorded to lifelong learning by the professional association, and the responsibility of its members to uphold a 'professional standard of care'. For members, the policy offers some legitimation in designating intentional learning time in their work schedules, and may motivate their capacity to seek resources and plan learning. The problem with the model is that it cannot indicate the nature and depth of engagement in learning, or the actual outcomes of engagement in terms of personal understandings and changes to practice. The learning events are not necessarily connected in any meaningful way with the actual contexts and dilemmas of a professional's practice. Nor does the model illuminate relative benefit of different activities in terms of professional learning, or offer recognition for learning within problems and relations of everyday practice.

\section{Problems of Self-Assessment and Their Learning Assumptions}


These three approaches to assessing lifelong learning - growth plans, self-assessment checklists, and learning event logs - are common models used for regulatory as well as developmental purposes in a range of professions in health, education and social service (e.g. Austin et al., 2008; Guskey, 2000; Imogen et al. 1999, Weddle et al., 2002). However, all three approaches are rooted in concepts of learning as an individual, psychological phenomenon, a process of 'acquiring' and perhaps reflecting upon new knowledge and skills. As argued in the following paragraphs, these concepts - and the assessment tools they produce - can constrain and distort professional learning.

\section{Knowledge as individually acquired}

Acquisition implies that knowledge is a pre-existing substance ingested by the learning individual and then applied to practice. Yet a general turn to practice-based perspectives of workplace learning discussed further on has generated wide acceptance that knowledge is embedded in everyday action, not in heads or even in bodies as dislocated skills. To view learning as limited to an individual consciousness 'acquiring' new knowledge and then 'carrying' it across time and space is to ignore growing evidence that knowledge is enacted and improvised within situational relations (Hager, 2004). Professionals collectively construct, modify, resist, and select different meanings of knowledge within the complex dilemmas of the everyday. Recent research on workplace learning has shown how different workplace environments produce learning (Billett, 2004; Fuller and Unwin, 2004). That is, particular spatiotemporal arrangements and discourses of work invoke particular practices, subjectivities and knowledge (Hearn and Michelson, 2006; Edwards and Nicoll, 2004; Fenwick, 2007).

Critics also have shown that a-political approaches to workplace learning tend to obscure the important power relations and hierarchies that determine what learning is most valuable, what counts as skill, and what knowledge remains marginal or unnamed (Farrell and Fenwick, 2007; Sawchuk et al. 2006). Power relations among professional disciplines and organizational sectors configure arrangements of activity and social divisions that enable some kinds of learning for some people, and constrain many others. Accounts and assessments of professional learning that are not integrated with these social, cultural and political dynamics construct knowledge and practice in problematic ways. Learning event logs, for example, separate learning from doing, knowledge-acquisition from knowledge application, and individual from collective. Even strong proponents of approaches such as professional portfolios have cautioned against the 'traps' of using these to assess learning (Boud, 1999).

\section{Reflective practice}

Second, the emphasis on reflective practice upheld in assessment practices such as growth plans and learning logs relies upon individual mentalist recall and disclosure of experiences - translated as learning. While reflection has been demonstrated useful for encouraging personal sensemaking in professional learning, formal reflective technologies such as supervised growth plans tend to overemphasise and under-theorise the role of individual reflection in professional knowledge. Experience is cast as static and sedimented, separated from knowledge-making processes. What is foregrounded are individual mental representations of events, disembodied, static and separated from the interdependent commotion of people together in action with objects and language.

Further, what people reflect upon and report as their learning may not correspond with how they actually participate in learning events and everyday practice. For seasoned 
professionals, much practice functions at a tacit level beyond conscious apprehension and language. Indeed when they are asked to trace their participation, it becomes clear that this tracing is actually a narrative performance that varies according to their interest, sense of comfort, familiarity with context and participants, the tools at hand, judgment of 'worthiness' of learning, etc. Little of what is actually emerging in the everyday processes of knowledge-making in work is visible to individual participants. As Lather (2000) wrote, 'What we think we see, when we reflect on experience 'is always already distorted .. a spectacle of replication in an excess of intention' (p. 154). Growth plans, for example, were not able to demonstrate specific improvements in practice, nor to represent the connections among different teachers' learning or the multiple webs of their collective activity, environments and their learning. Growth plans also measured learning in ways that contained professional knowledge in a September to June box, predictable, controllable, and documentable.

\section{Self-assessment}

Third, in terms of self-assessment, abundant research particularly in the health professions has demonstrated that self-ratings are problematic (Hodges et al., 2001; Kruger and Dunning, 1999; Regehr and Eva, 2007). Individuals' self-assessment bears little correlation with external assessments of those individuals. Most individuals overestimate their own performance in specific areas of practice, sometimes dramatically. The 'poorest performers' in particular tend to have difficulty discerning the difference between their practice and minimum standards of competence, even when shown examples of both. Kruger and Dunning (1999), among others, have argued that this is because low professional competence is partly due to inability to understand both what is required and the outcomes of one's own decisions and actions. That is, some professionals just cannot comprehend what they are doing wrong despite intensive assistance. On the other hand, it is very difficult for anyone to develop awareness of what one does not know. Furthermore, the judgment of low competence presumes a decidable performance requirement which may allow too little range for professional variation or, indeed, deliberate resistance to particular performance requirements.

Regehr and Eva (2007) argue that the problem lies in the conceptualization of selfassessment. Conventionally, self-assessment involves reflection removed from practice, attempting to determine one's general strengths and weaknesses overall. Instead, Regehr and Eva suggest assessments based on 'situation-specific self-awareness' ( $p$. S82) where professionals are encouraged to observe how they approach specific problems right in the heat of practice: where they experience uncertainty, what knowledge they seek out and where, whether they know when to defer the problem to other specialists and to slow down 'at the borders of competence' (p. S83). Overall, self-assessment in professional learning has been characterised by wide-ranging meanings, purposes and methodological problems that have raised calls for its complete reconceptualization (Ward et al., 2002).

\section{Reflexivity}

Finally, in all of these assessment technologies and indeed the whole enterprise of professional assessment, an important question lurks: who is really doing the assessment, and for what purpose? Who is looking at the growth plan or test results or learning log, and what meaning is being made of it? What knowledge is being recognised and for what purposes? What institutional, discursive and social conditions are being recognised as influencing how 
professionals are permitted to think and act? To what extent are learning encounters recognised to be sites of struggle in these conditions?

Assessment technologies shape how people come to think about their practice through disciplines of self-regulation and codification, as Foucault showed. The teachers in the TPGP study for example were quite clear about the dilemmas of risk posed by being compelled to pronounce and make visible certain weaknesses in their practice in order to comply with the annual standards for professional competence. More fundamentally, these technologies render multi-faceted complex experience into text. This moves something dynamic and ineffable into something fixed, decidable and visible, foreclosing the openings and the questions. As McWilliam (2002) points out, this move works to construct a limited view of what is worthwhile professional knowledge, and curtails debate on important contestations over knowledge and learning.

These issues are part of a larger debate about how to conceptualise and assess professionals' learning in ways that honor the complexities of practice and expertise, and that acknowledge more fully the important connectivities: among different learning actors; among actors and the tools, routines and architectures structuring their work; among actors and the institutions of professional associations, workplace and academic disciplinary knowledge; and among actors and the emerging collective knowledge and action in which they are enmeshed.

\section{Alternate Perspectives for Understanding Professional Learning}

Recent scholarship in work learning tends to accept that the learning process is distributed - that is, it is simultaneously both individual and collective, and that it cannot be valued apart from the contexts with which it is mutually constitutive: everyday action, planning, conversation, projects, problem-solving, instruction, reading, and online activity (Hager, 2004; Bratton et al., 2003; Sawchuk, et al. 2006). Amidst this scholarship increasing emphasis has been accorded to objects, to the material dimensions of learning and knowledge circulation, with the recognition that workplace practice represents a commingling of human and nonhuman entities that cannot be understood through analyses limited to social or constructivist perspectives of knowledge. Three perspectives that each take up this socio-material approach to analysis have been selected for discussion here: complexity science, cultural-historical activity theory, and actor-network theory. Unlike other perspectives of workplace learning that recognize distributed knowledge production and the importance of context, these three are unique in that they grant particular importance to the force exercised by nonhuman objects and artefacts in knowledge production. They share concern for how learning emerges as individuals and objects interact to enact what appear to be contextual structures and culture, the configurations of practice, and knowledge. Each is rooted in different, often contested, positions about the nature of knowledge, the nature of being, the relation of learner to object, and the nature of practice. Some try to totalise the debate. But their various contributions suggest fruitful openings for reconsidering processes through which learning in work adapts, expands and changes.

\section{Learning as emergence of collective cognition and environment: complexity science}

Complexity science provides one approach to understanding learning processes in a socio-cultural system such as a community of professionals (Davis and Sumara, 2006; Karpiak, 2000; Osberg and Biesta, 2007; Stacey, 2005). The key theme is emergence, the understanding 
that in (complex adaptive) systems, phenomena, events, environment and actors are mutually dependent, mutually constitutive, and actually emerge together in dynamic structures. Learning is defined as expanded possibilities for action, or becoming 'capable of more sophisticated, more flexible, more creative action' (Davis and Sumara, 2006).

In work organisations, people constantly influence and adjust to each other's emerging behaviours, ideas, and intentions - as well as with objects, furniture, technologies, etc - through myriad complex interactions and fluctuations. No clear lines of causation can be traced from these interactions to their outcomes, because at any given time among all these interconnections, possibilities are present in the system that are not visible or realised. New possibilities for action are constantly emerging among these interactions. Out of these continuous and non-linear interactions emerge dynamic structures that exceed their parts. Osberg and Biesta (2007) call this 'strong emergence': conditions where the knowledge and capability that emerges is more than the sum of its parts, and therefore not predictable from the 'ground' it emerges from. Knowledge and action are understood as continuous invention and exploration, produced through relations among consciousness, identity, action and interaction, objects and structural dynamics. Knowledge or skill cannot be contained in any one element or dimension of a system, for knowledge is constantly emerging and spilling into other systems. This means that humans are fully nested within and interconnected with many elements of the systems comprising them and in which they participate. They are not autonomous, sovereign agents for whom knowledge can be acquired or extracted. Rather, they perform themselves into existence.

\section{Learning as expansion of objects and ideas: cultural-historical activity theory}

Cultural-historical activity theory (CHAT) is increasingly used to understand how learning and activity are embedded in the interconnections of a workplace system(s) (Fuller and Unwin, 2004; Engeström, 2001; Sawchuk et al., 2006). First it is important to understand that actual and possible action in an activity system is shaped by its 'object', the problem at which activity is directed. The everyday action of work and learning is further shaped by the system's division of labour, community relationships, rules, tools and cultural norms as well as the different perspectives of the actors within it. CHAT theorists look carefully at the system's culture and its history - how things came to be as they are, and came to be viewed in ways that they do. They also focus on the contradictions that all systems carry within them. For example, many professionals' work organizations carry simultaneous pressures to innovate and take risks while performing with excellence, mastery and no error. Or, professionals' organizations require collaborative, interdisciplinary work and espouse collective knowledge while rewards and structures continue to focus on the efforts and competency of the autonomous individual.

From a CHAT perspective, learning is viewed as expansion of the system's 'object' and reconfiguration of the system's practices. Further, learning combines collective expansion and innovation with individual expansion in conceptions, interactions and practices. The expansion often comes about through the successive exacerbation and resolution of contradictions within the system. For example, within an organization that promotes collaborative work but gives most rewards to individual effort, some people might begin to seriously question the contradictions at play and their consequences. In a research-intensive university department, these questions could ultimately become directed at the overall 'object' driving their research. Is the object more to generate refereed journal publications and grants, or more to create networked relationships and to impact practice, if these two directions come into conflict? Is the object measured through visible short-term outcomes in place of ambiguous and unpredictable long-term outcomes, even 
when these may be more salient to deeper-impact research? Such questions, when taken up seriously throughout the organization, cause the object to expand and shift as individuals' understandings expand and shift. Learning thus occurs through a non-linear cycle of questioning something in this activity system, analysing its causes, modeling a new explanation or solution, implementing this model in the system, reflecting on it and consolidating it (Engeström, 2001). Much back-and-forth activity revolves around finding consensus about what exactly is the problem, and what can be tolerated as a solution or innovation within the politics of the system.

\section{Learning as 'translation' and mobilization: actor network theory}

A third perspective that departs from individual, psychologised notions of learning to examine how learning emerges in the relations and action of a system is actor network theory. Like complexity science, ANT is a diffuse series of perspectives, not one unitary conception (Latour, 2005), with roots in critical post structuralism. It explains that any changes we might describe as learning -- new ideas, innovations, changes in behavior, transformation - emerge through networks spread across time and space. These networks as well as the actors that they create are brought into existence through myriad negotiations among humans and non-human entities. ANT has been particularly helpful in analysing professionals' learning in these networks in studies of management learning (Fox, 2005), learning with ICTs (Edwards and Nicoll, 2006), and teachers' learning (Mulcahy, 2007). In one study using ANT, Gherardi and Nicolini (2000) examine how workers learned safety skills by tracking the negotiations of knowledge at every point as it moved through a system. For example, one workman would show another how to adapt a new safety procedure to make a task easier, or two together would adapt a particular tool to solve a problem, depending on who was watching. At other points in the system, the crew foreman negotiated the language of the safety assessment report with the industrial inspector. Deadlines and weather conditions caused different safety knowledge to be performed and different standards of evaluation. The equipment itself, and the crew's culture, embedded or 'grounded' a history of use possibilities and constraints that influenced the safety skills performed by those who interacted with the equipment. No skill or knowledge had a recognizable existence outside its use within the community.

Each entity in this process becomes an actor only when it succeeds in translating another actor, mobilising it to perform knowledge in a particular way, such as a worker translating a foreman into a disciplinarian through a particular set of behaviors. Eventually these dynamic attempts by actors to translate one another become stabilised: the network settles into a stable process or object that maintains itself. Like a black box it appears immutable and inevitable, while concealing all the negotiations that brought it into existence. An example would be a mandated list of professional competencies, or a so-called evidence-based practice accepted as 'gold standard'. Each entity also belongs to other networks in which it is called to act differently, taking on different shapes and capacities. A written contract, for example, is a technology that embeds knowledge, both from networks that produced it and networks that have established its use possibilities and constraints. In any employment arrangement the contract can be ignored, manipulated in various ways, or ascribed different forms of power. Thus, no agent or knowledge has an essential existence outside a given network: nothing is given in the order of things, but performs itself into existence. 'Moments' of translation have been framed (e.g. by Callon, 1986) as problematisation (where something tries to establish itself as an 'obligatory passage point' that frames an idea, intermediary or problem and related entities in particular ways); interessement (where selected separate entities are actively connected to this framing and where they negotiate 
their role in the emerging network); enrolment (the process whereby these entities become engaged in new identities and behaviours and increasingly 'translated' in particular directions by the network relations); and mobilisation (where the network is sufficiently durable that it can be extended to other locations and domains). As Fox (2005) explains, professional competence from an ANT perspective is not a latent attribute of any one element or individual, but a property of some actions rather than others as a network becomes enacted into being. This process of enactment, this interplay of force relations among technology, objects and changes in knowledge at every point in the network, is a continuing struggle - and this struggle is learning.

Each of the three socio-material perspectives presented here offers something unique to the conception of professional learning. Complexity science in its educational applications has emphasised the biological dimensions of learning, continuous adaptation across the system, the myriad interactions of diverse elements that give rise to many simultaneous creative experiments as well as disturbances in the system, that ultimately produce new knowledge and change. Cultural-historical activity theory focuses upon the history and power relations that determine what cultural practices came into being in a system, the mediation of these through system artefacts, and the object or problem at which activity is directed. CHAT emphasises the contradictions carried within the system, showing how when these are questioned with sufficient intensity, learning occurs: practices undergo expansion, and 'objects' become reframed. Actornetwork approaches go the furthest to insist on the symmetry of non-human and human participants, and focus on the micro-interactions and translations among these that assemble them into networks, mobilise particular behaviours and identities within these networks, and eventually stabilise them into black boxes of routines and beliefs. For complexity science, learning and change is about emergence of new unpredictable possibilities and forms. For CHAT enthusiasts, learning and change is about expansion of practices and perceptions. In ANToriented writings, notions of learning and change are related to translation of individual elements into networks of action and knowledge, and the resistances to translation.

\section{Discussion and Implications for Assessing Professional Learning}

What do any of these perspectives suggest for assessment practice? A full discussion of methodological implications lies beyond this discussion. This is mainly because the intent here was first to introduce and contrast socio-material theories for those who may be intrigued to follow up with their own study, and second to highlight the questions that each offers to conceptions of professional learning and its assessment. These theories each engage a complex rethinking of practice that cannot be - should not be - reduced to a toolbox. It may be best to consider each perspective as offering a different sensibility that can radically alter the way we view knowledge and its development. Within this sensibility, each may suggest possible approaches to practice, which will be outlined briefly in the following discussion.

Socio-material perspectives of knowledge urge a refocusing on the relations between things, not the things themselves, to observe complex wholes emerge from improvisations among micro-elements. Assessment approaches therefore, following educational research approaches crafted from principles of complexity adaptive systems (David and Sumara, 2006), would begin by tracking emerging patterns in a system, drawing particular attention to what occurs in the 'background': the myriad fluctuations, subtle interactions, the series of consequences emerging from a single action. Assessment would mimic the feedback loops of 
complex adaptive systems, providing feedback at various points in time and within system exchanges, detecting where bottlenecks, reduction of variation, or control of information are preventing emergence. From a complexity science perspective, feedback also focuses on disturbances, amplifying those with generative potential and highlighting those with destructive potential. A key element of assessment would be attuning participants in a complex system to its diversities, emerging patterns and dynamic structures, and helping them to assess these patterns and develop a 'complexified awareness' of their own and others' impacts on the larger system (Davis and Sumara, 2006). Finally complexity science would require any observer such as evaluators to also assess their own entangled involvements in the emerging systems of thought and action through the very process of assessment.

Complexity-informed assessment, like its research, is above all participatory and emergent. Approaches to such assessment can be borrowed from participatory research methods, which are well-known in the field of professional development. One approach would engage professionals in framing for themselves a collective dilemma of practice, then exploring, designing, acting and tracking what emerges through their intentional intervention. Participatory assessment, in particular, would focus on collective identification of benchmarks or snapshots of practice that can be compared at different stages, flexible indicators from different perspectives to help characterise what is emerging and what count as outcomes, and language to describe processes, strategies and uncertainties. Participatory assessment also would capture and compare individuals' perceptions, assumptions and judgments about the process and its outcomes over time.

While CHAT theorists haven't specified measurement protocols for such learning, we can extrapolate some questions from its principles that might be raised in attempts to assess professionals' learning. Engeström (1999, p. 384) explains learning as 'expansion' of object and practice, through the 'construction and resolution of successively evolving tensions or contradictions in a complex system that includes the object or objects, the mediating artifacts, and the perspectives of the participants.' Assessment logically would begin by establishing the dynamics of tools, community, divisions of labour, and rules interacting with the professional in everyday activity. Questions would examine how the collective object, and the individual professional's object of focus, emerged and whether it is being redefined. In terms of learning, assessment would explore how this object(s) might expand, and whether and how it is currently undergoing configuration. Further, where are the internal contradictions held within this system (among objects, tools, divisions of labor, boundaries, etc)? What new tensions are emerging? What internal tensions could stimulate change? (Which ones could be articulated?) What boundaries prevent expansion?

In ANT-influenced analyses of workplace knowledge, questions are posed that could guide new ways to approach assessment. For example, how do the material objects of the practice configure professionals' action and response? Immediately the focus shifts from the individual 'learner' to the negotiations among human and nonhuman entities that enact knowledge. ANT sensibilities go on to ask: How do the networks that function as knowledge 'actors' (particular practices, standards, codified professional knowledge) emerge? What connections assemble objects and professionals into the networks that become these actors? What and who becomes included and excluded? What individual identities, behaviors have been translated in becoming part of the network? What negotiations occur as individual elements take up, resist, or compete with the attempts to enroll and mobilise them into particular patterns of action and knowledge? Working from such questions, the assessment of professional learning 
then becomes a process of tracing how knowledge circulates - both among humans and nonhumans within particular contexts, and across institutions enmeshed in determining and regulating professionals - to enroll, mobilise and to stabilise particular practices and ideas. The approaches employed to address such questions are necessarily messy (Law, 2004), and firmly resist being transformed into the tidy technical tools that evaluators might desire. But this is precisely the point. Until assessment approaches are willing to engage with the ontological dilemmas that are really at stake in the socio-material ways that professional practices are configured and reconfigured, and the ways that individual humans and their conceptions and what appears to be their competencies are intimately entwined in these webs, they will continue to yield little more than a reflection of themselves.

\section{Conclusion}

This discussion has contended that conventional approaches to understanding and assessing professionals' learning, such as reflective growth plans or portfolios, self-administered tests and learning event logs, embed several problems of validity and scope. These problems are related to psychologised and individualist assumptions that professionals' learning is predominantly acquisitive, a-political, conscious and representable. The position argued here is that richer approaches to learning assessment could be derived from practice-based socio-material perspectives that have been applied by organizational theorists to analyse knowledge, learning and change in work environments. Three perspectives were outlined: complexity science, cultural-historical activity theory and actor network theory. Each of these perspectives proposes different questions for evaluation. These questions open possibilities for considering the enmeshment of professionals' learning in the webs of collective action and cultural discourses, nodes of micro-negotiation and struggle, politics of knowledge and institutions, and systemic contradictions of their work. Further, these questions unsettle one another and highlight their own and each other's limitations. These questions also fold the gaze of assessment back to itself. No position of observance or representation is possible outside the webs of collective action. All questions and tools of assessment are seeds of change feeding into the system; the evaluator, the educator and the learner are entangled and mutually constitutive in the emerging knowledge.

What gets measured is what gets learned, goes the old saw. While learning is far more dynamic than this might imply, it is clear that assessment technologies as a form of representation powerfully shape practice or at least individuals' sense of what is valued in practice. This is the making of practice according to measure. Too many measures of professionals' learning are distant from practice, overly determined, reductionist, and nonreflexive about their limitations and their performative power. Under their regulation, professional knowledge as well as professional subjectivities can become truncated or misdirected. If educators and evaluators genuinely seek change in professionals' approach to learning, we surely must be willing to begin by seeking alternate approaches to assessment.

A first step might be to expand our own fundamental understandings of learning, to recognize the distributed nature of workplace learning and to begin to appreciate the central role of materiality and the force of objects in workplace enactments of practice and knowledge. The pragmatic short-term approach may lie in not just in adopting multiple methods of assessment, but also in interrupting and opening out our basic assumptions about what comprises professional learning. Educators already know that good evaluation practice depends upon a mix 
of approaches to provide different forms of information and raise different questions. The opportunity now is to recognise the limitations of these approaches as well as their contributions, to foreground their technologies, politics and assumptions about learning, and to explore alternate conceptions that can shed light on knowledge emergence from a socio-material perspective.

\section{References}

Alberta Learning. 1998 . Teacher growth, supervision, and evaluation, January 26, Policy 2.1.5 Alberta College of Pharmacists. 2008 . Continuing competence. Retrieved on January 312008 from https://pharmacists.ab.ca/nContinuingCompetence/default.aspx

Austin, Z., Gregory, Paul A.M., Galli, M. 2008 . 'I just don't know what I'm supposed to know' Evaluating self-assessment skills of international pharmacy graduates in Canada. Resarch in Social and Administrative Pharmacy 4 (2): 115-24.

Fenwick, T. (2003a). The good teacher in neo-liberal risk society: A Foucaultian analysis of professional growth plans. Journal of Curriculum Studies, 35 (3), 335-354

Fenwick, T. (2004). Teacher learning and professional growth plans: Implementation of a provincial policy. Journal of Curriculum and Supervision, 19 (3), 259-282.

Billett S. 2004 . Co-participation at work: Learning through work and throughout working lives. Studies in the Education of Adults. 36(2):190-205

Boud, D. 1999. Avoiding the traps: Seeking good practice in the use of self assessment and reflection in professional courses. Social Work Education, 18 (2): 121-132

Bratton, J.,Mills, J.H.,Pyrch, T., and Sawchuk, P. 2003. Workplace learning: a critical introduction. Aurora, ON: Garamond Press.

Callon, M. 1986 . Some elements of a sociology of translation: domestication of the scallops and the fishermen of St Brieuc Bay. In: J.Law (ed.) Power, Action and Belief. A New Sociology of Knowledge?, pp. 196-233. London: Routledge \& Kegan Paul.

CMA 2000 . Certified Management Accountants Regulation Part 1 Division 3, Section 15(a),(i). Continuous Professional Learning and Development Policy. Retrieved on February 21, 2008 from http://www.cma-alberta.com/8/4/8/5/index 1.shtml

Davis, B., and Sumara, D.J. 2006 . Complexity and education: inquiries into learning, teaching and research. Mahwah, NJ: Erlbaum.

Engeström, Y. 2001 . Expansive learning at work: Toward an activity theoretical reconceptualization. Journal of Education and Work 14 (1): 133-146.

Eraut, M. 1994. Developing professional knowledge and competence. London: Routledge.

Eraut, M. 2004. Informal learning in the workplace. Studies in Continuing Education, 26 (2), 247- 274

Evetts, J. 2003 . The sociological analysis of professionalism: Occupational change in a modern world. International Sociology 18 (2): 395-415.

Farrell, L. and Fenwick, T. (2007). Educating the global workforce? In L. Farrell and T. Fenwick (eds.) Educating the Global Workforce. London: Routledge.

Fenwick, T. (2003). The good teacher in neo-liberal risk society: A Foucaultian analysis of professional growth plans. Journal of Curriculum Studies, 35 (3), 335-354 
Fenwick, T. (2007). Escaping/becoming subjects: learning to work the boundaries in boundaryless work. In S. Billet, T. Fenwick \& M. Somerville, Work, learning and subjectivity. New York: Springer.

Fenwick, T. and Edwards, R. (forthcoming). Actor-network theory in education. London: Routledge.

Fox, S. 2005 . An actor-network critique of community in higher education: Implications for networked learning. Studies in Higher Education, 30 (1): 95 - 110

Fuller, A. and Unwin, L. 2004 . Expansive learning environments: Integrating organizational and personal development. In H. Rainbird, A. Fuller and A. Munro, (Eds.) Workplace learning in context (pp. 126-144). London: Routledge.

Gherardi, S. and Nicolini, D. 2000 . To transfer is to transform: The circulation of safety knowledge. Organization 7 (2): 329-348.

Guskey, T.R 2000 . Evaluating professional development. Corwin Press.

Hager, P. 2004 . Lifelong learning in the workplace? Challenges and issues. Journal of Workplace Learning, 16 (1/2): 22-33.

Hearn, M. and Michelson, G. 2006 . Rethinking work: Time, space and discourse. Cambridge: Cambridge University Press.

Hodges B, Regehr G, and Martin D. 2001. Difficulties in recognizing one's own incompetence: Doctors who are unskilled and unaware of it. Academic Medicine, 76: S87-S89.

Imogen, T. Thomas, J. \& Sage,H. 1999. Portfolios for learning and assessment: Laying the foundations for continuing professional development, 18(2): 147-160.

Kruger J and Dunning D. 1999. Unskilled and unaware of it: How difficulties

in recognizing one's own incompetence lead to inflated self-assessments. Journal of Personnel and Social Psychology, 77, 1121-1134.

Latour, B. 2005. Re-assembling the social - An introduction to actor network theory. London: Oxford University Press.

McWilliam, Erica L 2002 Against professional development. Educational Philosophy and Theory 34(3): 289-300.

Mulcahy, D. 2007 . (Re)working relations of strategy and spatiality in education. Studies in Continuing Education, 29 (2): 143-162.

Osberg, D. and Biesta, G.J.J. 2007 . Beyond presence: epistemological and pedagogical implications of 'strong' emergence. Interchange, 38(1), 31-51.

Personal communication 2008 . Dr. Terri Schindel, Director of Continuing Pharmacy Education University of Alberta, February 6, 2008.

Regehr, G. and Eva, K. W. 2007. Knowing when to look it up: a new conception of selfassessment ability. Academic Medicine, 82 (10).

Sawchuk, P., Duarte, N., and Elhammoumi, M. 2006 . Critical perspectives on activity: explorations across education, work, and everyday life. Cambridge University Press.

Stacey, R.D. 2005 . Experiencing emergence in organizations: local interaction and the emergence of global pattern. London: Routledge.

Stronach, I., Corbin, B., McNamara, O., Stark, S. and Warne, T. 2002 . Towards

an uncertain politics of professionalism: teacher and nurse identities in flux. Journal of Education Policy, 27 (2): 109-138.

Ward, M., Gruppen, L. and Regehr, G. 2002 . Measuring self-assessment: current state of the art. Advances in Health Sciences Education 7: 63-80. 
Weddle, D., Himburg S.P., Collins N. and Lewis R. 2002. The professional development portfolio process: Setting goals for credentialing. Journal of the American Dietetic Association, 102 (10): 1439 - 1444 